OPEN ACCESS

Edited by:

Adriana Ximenes-da-Silva,

Federal University of Alagoas, Brazil

Reviewed by:

Susanna Scafidi,

Johns Hopkins University,

United States

Nafisa M. Jadavii,

Ottawa Hospital Research

Institute (OHRI), Canada

*Correspondence:

Michelly Pires Queiroz

queiroz_m.p@hotmail.com

Juliana Késsia Barbosa Soares

julianakessia2@gmail.com

Specialty section:

This article was submitted to Neuroenergetics, Nutrition and Brain

Health,

a section of the journal

Frontiers in Neuroscience

Received: 06 September 2018

Accepted: 01 April 2019

Published: 24 April 2019

Citation:

Queiroz MP, Lima MS,

Barbosa MQ, Melo MFFT, Bertozzo CCMS, Oliveira MEG, Bessa RJB, Alves SPA, Souza MIA,

Queiroga RCRE and Soares JKB (2019) Effect of Conjugated Linoleic Acid on Memory and Reflex Maturation in Rats Treated During Early Life. Front. Neurosci. 13:370. doi: 10.3389/fnins.2019.00370

\section{Effect of Conjugated Linoleic Acid on Memory and Reflex Maturation in Rats Treated During Early Life}

\author{
Michelly Pires Queiroz ${ }^{1 *}$, Martiniano da Silva Lima², Mayara Queiroga Barbosa², \\ Marilia Ferreira Frazão Tavares de Melo², \\ Camila Carolina de Menezes Santos Bertozzo2, Maria Elieidy Gomes de Oliveira 1,2, \\ Rui José Branquinho Bessa ${ }^{3}$, Susana Paula Almeida Alves', \\ Maria Izabel Amaral Souza ${ }^{4}$, Rita de Cassia Ramos do Egypto Queiroga ${ }^{1,5}$ and \\ Juliana Késsia Barbosa Soares ${ }^{1,2 *}$

\footnotetext{
${ }^{1}$ Program of Food Science and Technology, Federal University of Paraíba, João Pessoa, Brazil, ${ }^{2}$ Laboratory of Experimental Nutrition, Department of Nutrition, Federal University of Campina Grande, Campina Grande, Brazil, ${ }^{3}$ Centre for Interdisciplinary Research in Animal Health (CIISA), Faculty of Veterinary Medicine, University of Lisbon, Lisbon, Portugal, ${ }^{4}$ Program in Animal Science, School of Veterinary and Animal Science, Federal University of Goiás, Goiânia, Brazil, ${ }^{5}$ Laboratory of Bromatology, Department of Nutrition, Federal University of Paraíba, João Pessoa, Brazil
}

In the critical period of neurodevelopment (gestation and lactation), maternal consumption of essential fatty acids (FAs) can alter the offspring cognitive function permanently causing damage. Lipids can regulate neurotrophin and compose brain tissue. However, the effects of maternal consumption of a mixture of conjugated linoleic acid (CLA) on an offspring nervous system are not completely clear. We aimed to investigate the impacts of different CLA concentrations mixed into the maternal diet during early life on neonatal reflex maturation and cognitive functions of the offspring. Three groups were formed: control (CG): receiving a standard diet; CLA1: receiving a diet containing $1 \%$ of CLA, and CLA3: receiving a diet containing 3\% of CLA, offered during gestation and lactation. After birth, the reflex responses of the offspring were observed from the 1st to the 21 st day. After weaning, the animals' anxiety and memory were assessed using open field (OF) and novel object recognition tests. Fatty acids in the breast milk and the offspring's brain were also quantified. The data were analyzed using one-way ANOVA and the Kruskal-Wallis test. CLA1 presented accelerated palmar grasp disappearance versus CLA3 and negative-geotaxis versus CG; and the CLA3 presented increases for most reflexes (cliff-avoidance, vibrissa-placing, negative-geotaxis, and auditory-startle response), and decrease in reflexes palmar grasp and free-fall righting versus CG $(p<0.05)$. CLA3 group explored less of the OF in the second exposure. CLA1 and CLA3 presented an increased exploration ratio for new objects, which indicates memory improvement. The milk tested from CLA3 demonstrated an increase in polyunsaturated fatty acids (PUFAs), and a decrease in monounsaturated fatty acids. The amount of CLA in milk was greater in CLA1 and CLA3 and in the brain offspring both presented moderated amounts of CLA. Maternal treatment with the CLA mixture induced anticipated reflex maturation and improved memory in the offspring. Even 
though CLA was detected in the brains in only trace amounts, offspring's brain PUFA and SFA levels were increased. Further studies aimed to delineate the effect of maternal CLA supplementation on offspring's brain lipid metabolism and long-term neurologic outcome are needed to confirm these findings.

Keywords: conjugated linoleic acid, neurodevelopment, reflex maturation, memory, physical parameters, fatty acids, maternal nutrition

\section{INTRODUCTION}

The central nervous system first appears in the human embryo at around the 3rd or 4th week after fertilization; development continues until roughly to 2 years of age. In rats, development occurs from the second week of pregnancy until the end of lactation. This phase is known as "the critical period of development" and any injury can cause permanent damage (Morgane et al., 1993; Hsieh and Brenna, 2009).

In this critical period of development, there is an increased need for polyunsaturated fatty acids (PUFAs) in brain; chiefly arachidonic acid (AA, 20:4n-6) and docosahexaenoic acid (DHA, $22: 6 n-3)$, which together comprise about $20 \%$ of the brain tissue (Valenzuela and Nieto, 2003). DHA provides better blood flow and optimizes the development and functions of the neuronal membrane (Valenzuela and Nieto, 2003; Balogun et al., 2014). Regular PUFA intake is important for neurotrophin regulation. Neurotrophins perform essential functions during the development of the fetal nervous system (Balogun et al., 2014). During lactation, breast milk replaces the placental function by carrying nutrients from the nursing mother to the neonate. The lipid fraction present in breast milk, in addition to its energy supply function, is also responsible for myelin sheath structuring.

Found in breast milk, linoleic (18:2n-6) and linolenic (18:3n3) are PUFAs, and also essential fatty acids (FAs). Adequate intake of FAs is necessary for proper neurological and cognitive development in infants, and deficiencies during the brain development phase are associated with behavioral abnormalities (Hayat et al., 1999; Herrera, 2002; Gustavsson et al., 2010). Conjugated linoleic acids (CLAs) are a family of linoleic acid isomers presenting conjugated double bonds. CLAs are naturally produced by ruminant animals, found in their milk fats and muscle tissue, and in food products derived from them (Pariza et al., 2001; Banni, 2002). CLA isomers are commercially prepared by partial hydrogenation of linoleic acid (Banni, 2002). CLAs have been widely investigated due to their many beneficial health effects (Halade et al., 2010; Park et al., 2010; Furlan et al., 2013; Jelińska et al., 2014). It was found that CLAs cross the blood-brain barrier (Jelińska et al., 2014), inhibit angiogenesis in the mammalian brain (Sikorski et al., 2008), and in vitro were found to protect cortical cells against neurotoxic elements (Hunt et al., 2010). A maternal diet containing goat milk fat (as a source of CLAs), has also been found to affect cortical electrical activity (Soares et al., 2012) and anxiety in rats (Soares et al., 2013).

In the previous studies, goat milk was used as a source of CLA, but also of other lipids such as AA and DHA. Lipids of the n-3 series, present in goat's milk, are already known for their beneficial effects on the nervous system when supplemented during pregnancy and lactation. Research has found improvement in the memory of pups whose mothers were supplemented during pregnancy with this lipid and with valproic acid. This medication is used indiscriminately in pregnancy and can cause adverse effects, such as fetal malformation and cognitive defects (Chalon et al., 1998). Improvement in brain development has also observed in mice receiving series- 3 fatty acids (DHA, EPA, and AA) during lactation (Gaoa et al., 2016). However, studies analyzing the effects of CLA alone in these phases of life are still scarce.

It is well-known that dietary lipids, when offered during the initial phases of life, may alter reflexes, maturation (Santillan et al., 2010; Aquino et al., 2015), and behavior in animals (Soares et al., 2013). The hypothesis of this study is that maternal feed supplementation containing a mixture of CLA isomers during pregnancy and lactation positively influences reflex maturation (short term), and improves memory (long term) in rat offspring. This study aims to investigate the impact of supplementing the maternal diet with differing concentrations of a commercial CLA mixture on reflex ontogeny and memory in offspring.

\section{MATERIALS AND METHODS}

\section{Animals and Diets}

Female Wistar rats ( $n=12$, four female per each group), acquired from the Federal University of Paraíba (UFPB), aged 90 days and weighing $230 \pm 30 \mathrm{~g}$ were used to obtain pups ( $n=36$, only males were used). One female was maintained for each male during the mating period.

After confirmation of pregnancy, the mothers were housed in individual polypropylene maternity cages under standard conditions: temperature $22 \pm 1^{\circ} \mathrm{C}$, with a light-dark cycle $(12 \mathrm{~h}$; first light at 6:00 h), humidity of approximately $65 \%$, and food and water ad libitum. During the first week of gestation, the rats received a commercial diet (Presence Purina ${ }^{\circledR}$, São Paulo, Brazil), and an experimental diet was then offered starting from the second week of gestation and throughout lactation. During pregnancy and lactation, maternal feed intake and body weight were measured weekly. Three groups were formed: the control group (CG) receiving a standard diet without CLA $(n=11)$; the CLA1 group receiving an experimental diet containing $1 \%$ CLA $(n=13)$; and the CLA3 group receiving an experimental diet containing 3\% CLA $(n=12)$ (Table 1); all diets were in accordance with the recommendations of the American Institute of Nutrition (AIN-93G) (Reeves et al., 1993). The CLA mix used was Clarinol ${ }^{\circledR}$ powder (Stepan Lipid Nutrition, Maywood, NJ, United States); the composition is shown in Table 2. 
TABLE 1 | Composition of control and experimental diets.

\begin{tabular}{lccc}
\hline Ingredient (g/kg) & \multicolumn{3}{c}{ Diets } \\
\cline { 2 - 4 } & Control & CLA1 & CLA3 \\
\hline Corn starch & 530 & 520 & 500 \\
Casein & 199.5 & 199.5 & 199.5 \\
Sucrose & 100 & 100 & 100 \\
Soybean oil & 70 & 70 & 70 \\
CLA mix isomers & - & 10 & 30 \\
Fiber & 50 & 50 & 50 \\
Mineral mix & 35 & 35 & 35 \\
Vitamin mix & 10 & 10 & 10 \\
L-Cystine & 3.0 & 3.0 & 3.0 \\
Choline bitartrate & 2.5 & 2.5 & 2.5 \\
Gross energy (Joules) & $16.568,6$ & $16.777,8$ & $17.196,2$ \\
\hline
\end{tabular}

TABLE 2 | Fatty acid composition of the commercial CLA mix.

\begin{tabular}{lrc}
\hline Fatty acid & Mean & Standard deviation \\
\hline $14: 0$ & 0.1 & 0.011 \\
$16: 0$ & 4.3 & 0.038 \\
$18: 0$ & 1.4 & 0.021 \\
$18: 1 c 9$ & 10.7 & 0.135 \\
$18: 1 c 11$ & 0.6 & 0.024 \\
$18: 2 n-6$ & 1.0 & 0.027 \\
$20: 0$ & 0.2 & 0.014 \\
CLA-c9t11 & 39.2 & 0.097 \\
CLA-t10c12 & 38.3 & 0.071 \\
CLA c/t and t/c isomers & 2.5 & 0.128 \\
CLA-trans,trans & 1.5 & 0.042 \\
$22: 0$ & 0.2 & 0.010 \\
\hline
\end{tabular}

The litters were standardized with six pups and some parameters were evaluated: Litter size, Number of males, Number of females, Birth weights. After weaning, at 21 days of age, the animals were separated in polypropylene cages, two animals per cage, where they received water and commercial feed ad libitum, containing 1.589,9 J of energy, $23 \mathrm{~g}$ of proteins (24.21\%), $63 \mathrm{~g}$ of carbohydrates $(66.31 \%)$, and $4 \mathrm{~g}$ of lipids $(9.47 \%)$.

The research followed an experimental protocol in accordance with the ethical recommendations of the National Institutes of Health (Bethesda, MD, United States), and was approved by the ethics research committee of the Federal University of Paraíba No. 0407/13 (Figure 1).

\section{Physical Maturation}

The pups were weighed throughout lactation at 28, 49, and 70 days of life.

\section{Reflex Ontogeny in Newborn Pups}

From the 1st day through the 21st postnatal day, the reflex responses were observed each day at 12:00 pm. Response consolidation was considered positive when a reflex reaction was repeated for three consecutive days (Smart and Dobbing, 1971), have established an experimental model for reflex maturation in rats, as presented in Table 3.

\section{Open Field Habituation Test}

The open field habituation test is used to evaluate the animal to long-term habituation capacity on the open field device, consisting of a circular metallic arena (painted white) delimited by white walls with an open ceiling. The floor of the arena is divided into 17 fields (with lines painted black), 3 concentric circles $(15,34$, and $55 \mathrm{~cm}$ in diameter, respectively) which are subdivided into a total of 16 segments and a central circle.

In rodents, habituation is analyzed by locomotor activity on the open field and is considered an indicator of non-associative learning (Leussis and Bolivar, 2006; Rachetti et al., 2013).

At 42 days of age, the animals were exposed to the open field in two phases, the second exposure occurred seven (7) days after the first. Four parameters were evaluated in the first and second expositions, each during $10 \mathrm{~min}$ (Rachetti et al., 2013; Gamberini et al., 2015; Speight et al., 2017).

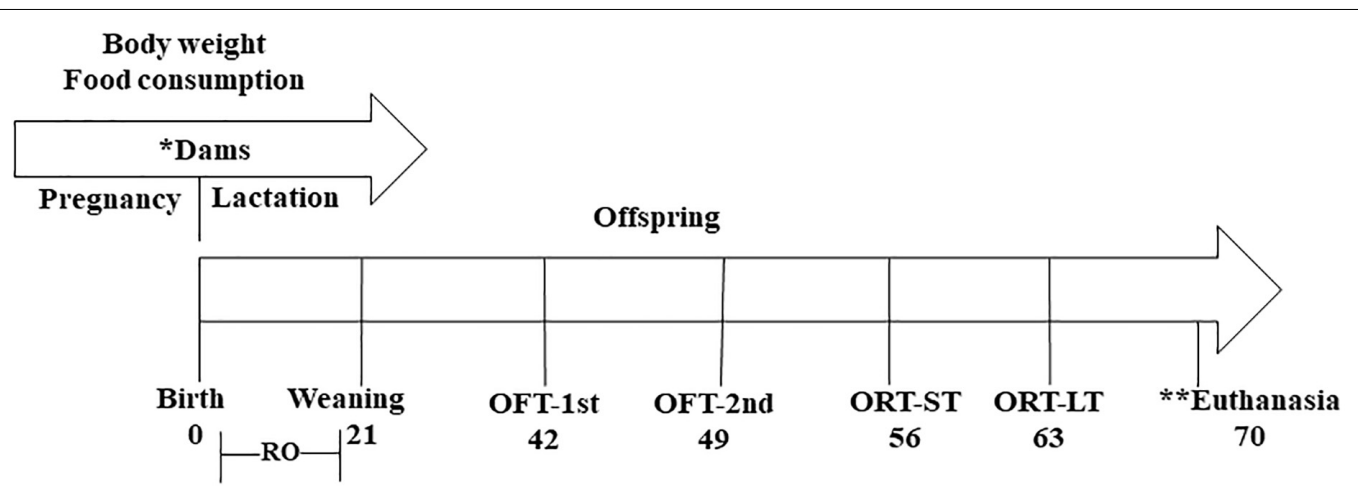

Days of life

FIGURE 1 | Experimental design. $\left(^{*}\right)$ Treatment period where the mothers received: control diet $(\mathrm{CG})(n=11)$; diet containing $1 \%(n=13)$; and diet containing $3 \%$ CLA $(n=12)$. $\left({ }^{* *}\right)$ Brain collection for fatty acid analysis. RO, reflex ontogeny; OFT-1st, open field test 1st exposure; OFT-2nd, open field test 2nd exposure; ORT-ST, object recognition test, short-term; ORT-LT, object recognition test, long-term. 
TABLE 3 | Description of the reflex test.

\begin{tabular}{|c|c|c|}
\hline Reflex & Stimulus & Response \\
\hline Palmar grasp & $\begin{array}{l}\text { Light percussion on the palm } \\
\text { of the right foreleg }\end{array}$ & Quick bending of ankles \\
\hline Righting & $\begin{array}{l}\text { The rat is placed in supine } \\
\text { position on a surface }\end{array}$ & $\begin{array}{l}\text { Return to the prone position } \\
\text { with all paws in } 10 \mathrm{~s}\end{array}$ \\
\hline Cliff-avoidance & $\begin{array}{l}\text { The rat is placed on a flat and } \\
\text { high surface (table), with legs } \\
\text { toward the extremity }\end{array}$ & $\begin{array}{l}\text { Moves to one side and walks } \\
\text { in the opposite direction to } \\
\text { the edge }\end{array}$ \\
\hline Vibrissa-placing & $\begin{array}{l}\text { The is was suspended by the } \\
\text { tail and its vibrissae lightly } \\
\text { touch the edge of a flat } \\
\text { surface }\end{array}$ & $\begin{array}{l}\text { Both front legs are placed on } \\
\text { the table, performing march } \\
\text { movements }\end{array}$ \\
\hline $\begin{array}{l}\text { Negative- } \\
\text { geotaxis }\end{array}$ & $\begin{array}{l}\text { The rat is placed at the center } \\
\text { of an inclined ramp with head } \\
\text { facing downwards }\end{array}$ & $\begin{array}{l}\text { Body spin at an angle of } \\
180^{\circ} \text {, positioning head } \\
\text { upwards }\end{array}$ \\
\hline $\begin{array}{l}\text { Auditory-startle } \\
\text { response }\end{array}$ & $\begin{array}{l}\text { Intense and sudden sound } \\
\text { stimulus }\end{array}$ & $\begin{array}{l}\text { Retraction of anterior and } \\
\text { posterior legs, with rapid and } \\
\text { involuntary body } \\
\text { immobilization }\end{array}$ \\
\hline Free-fall righting & $\begin{array}{l}\text { Held by the four legs, at a } \\
\text { height of } 30 \mathrm{~cm} \text {, it is released } \\
\text { in free fall on a synthetic foam } \\
\text { bed }\end{array}$ & $\begin{array}{l}\text { Position recovery during } \\
\text { freefall on the surface } \\
\text { supported on four paws }\end{array}$ \\
\hline
\end{tabular}

- Duration of locomotion - Time spent by the animal moving in the open field.

- Number of crossings in open field - The ambulation was evaluated by the total of segments covered. It was counted when the animal inserted the four legs inside the segments.

- Number of entries into inner zone - Number of entries into inner zone - Quantified when the animal placed the four legs inside each inner zone of the open field.

- Duration in inner zone - Time spent by the animal in the inner zone of the open field.

All of the sessions were recorded with a video camera attached to the laboratory ceiling and the videos were analyzed afterward. The videos containing the data were analyzed randomly and by a single evaluator.

\section{Object Recognition Test}

To evaluate declarative memory, when the animals reached 56 days of life, object recognition testing was applied on the previously used open field arena. Here, the animals underwent two open field exposures (the second exposure at 7 days after the first). The first test is associated with short-term memory, the second test relates to long-term memory (Rachetti et al., 2013).

The testing assesses the amount of time an animal spends in sniffing or touching an object with its nose and/or front legs. First, habituation is performed in the absence of any objects; animals may freely explore the arena for $3 \mathrm{~min}$. Next, in the training session, the animals are placed in the arena when containing two different objects (A1 and A2) allowing free exploration for $10 \mathrm{~min}$, for the animals to recognize and identify object A1 (a familiar object). The test session is held at $180 \mathrm{~min}$ after the training session to evaluate short-term memory, in which the animals are placed in the arena now containing two objects, A1 (the familiar object) and A3 (a new object), and they are allowed to freely explore for $5 \mathrm{~min}$. After 7 days, another test is performed to assess long-term memory, in which the animals are placed in the arena to freely explore object A1 (the familiar object) together with object A4 (a new object). The videos were subsequently analyzed by a single evaluator on a random basis, that is, the evaluator was not aware of which group was being evaluated.

Before and after each test, the device and the objects were cleaned with $10 \%$ alcohol, and when exchanging animals, both the device and objects were cleaned with 10\% alcohol and paper towels.

With completion of the test, the results obtained were analyzed using both the total time spent exploring the objects, and the novel object/(total familiar + novel object) ratio (Gustavsson et al., 2010).

\section{Profile of Brain and Milk Fatty Acids}

The milk was collected at the end of lactation (21th day after weaning). Oxytocin $(0,5 \mathrm{ml})$ was administered to facilitate lactation. For collection, the animals were anesthetized with ketamine hydrochloride and xilasin ( $1 \mathrm{ml} / \mathrm{kg}$ body weight). The milk was removed by hand (squeezing the rat's breast) and placed in encoded Eppendorf tubes, 3 per group, where 2 CG Eppendorf tubes and 1 Eppendorf tube for CLA3 were lost during the journey, leaving 1 sample of CG, 3 samples of CLA1 and 2 of CLA3. After collection, the rats were sacrificed by cervical detachment.

At the end of the experiment, at 70 days of age and after a 6-h fast, the offspring were anesthetized and sacrificed. After euthanasia, the brain was removed using a scalpel and pliers, and then lyophilized.

Milk and brain samples were sent to the Faculty of Veterinary Medicine at the University of Lisbon where the FA analyses were conducted. Fatty acid methyl esters (FAMEs) from the freezedried milk fat samples were prepared by direct trans-esterification using potassium hydroxide (2M) in methanol, in accordance with (Rego et al., 2009) and FAMEs and dimethyl acetal (DMA) from the brain samples were prepared by reaction with $\mathrm{HCl}$ $1.25 \mathrm{M}$ in methanol for $20 \mathrm{~h}$ at $50^{\circ} \mathrm{C}$. Fatty acid methyl esters and DMA were analyzed by gas chromatography with flame ionization detection using a Shimadzu GC 2010-Plus (Shimadzu, Kyoto, Japan) equipped with a SP-2560 (100 m $\times 0.25 \mathrm{~mm}$, $0.20 \mu \mathrm{m}$ film thickness, Supelco, Bellefonte, PA, United States) capillary column. The chromatographic conditions were as follows: injector and detector temperatures were set at 250 and $280^{\circ} \mathrm{C}$, respectively; helium was used as the carrier gas at $1 \mathrm{~mL} / \mathrm{min}$ constant flow; the initial oven temperature of $50^{\circ} \mathrm{C}$ was held for $1 \mathrm{~min}$, increased at $50^{\circ} \mathrm{C} / \mathrm{min}$ to $150^{\circ} \mathrm{C}$ and held for $20 \mathrm{~min}$; then increased at $1^{\circ} \mathrm{C} / \mathrm{min}$ to $190^{\circ} \mathrm{C}$; and finally increased at $2^{\circ} \mathrm{C} / \mathrm{min}$ to $220^{\circ} \mathrm{C}$ and held for $40 \mathrm{~min}$. Identification of FAME and DMA were achieved using electron impact mass spectrometry using a Shimadzu GC-MS QP2010 Plus (Shimadzu) and published chromatograms (Alves et al., 2013). The chromatographic column and the GC conditions were similar to the GC-FID analyses. Additional mass spectrometer conditions were as follows: ion source temperature, $200^{\circ} \mathrm{C}$; interface temperature, $240^{\circ} \mathrm{C}$; and emission voltage, $70 \mathrm{eV}$. The 
fatty acids and DMA inside the incubation tubes were expressed as milligrams per flask, and determined using the internal standard, assuming a direct proportionality between GC-FID peak area and FA weight.

\section{Statistical Analysis}

Intergroup differences for reflex maturation were analyzed using one-way Kruskal-Wallis testing, followed by Dunn testing on Sigma Stat software (San Jose, CA, United States). Milk sample FA profile data were presented as means and as standard deviations when more than one sample per treatment was available. Brain tissue FA profile data were analyzed using PROC MIXED, SAS 9.4 (SAS Inst., Cary, NC, United States) using a model that considered the treatment as a single fixed effect, and allowed for variance heterogeneity between treatments. When significant $(p<0.05)$ treatment effects were detected the least square means were compared using the Tukey procedure.

\section{RESULTS}

\section{Maternal Feed Intake and Pup Weights}

There were no significant differences in maternal food intake and body weight among the different groups.

Body weight analyses showed that the CLA3 group of pups presented higher body weights than the other two groups at 1, 14, and 21 days of age. However on day 7, the CLA3 group body weight was significantly higher only as compared to the CLA1 group, and not to the CG $(p<0.05)$. When assessing body weight after lactation, when animals were 28 days old, CLA3 had a higher body weight when compared to CLA1 and CG. At 49 days of age, the animals of the CLA1 and CLA3 groups presented higher body weights versus CG $(p<0.05)$. At the end of the experiment no significant statistical differences were observed between the groups (Figure 2).

\section{Birth Data}

Table 4 shows the parameters evaluated after birth. The CLA1 and CLA3 groups presented larger litters as compared to CG

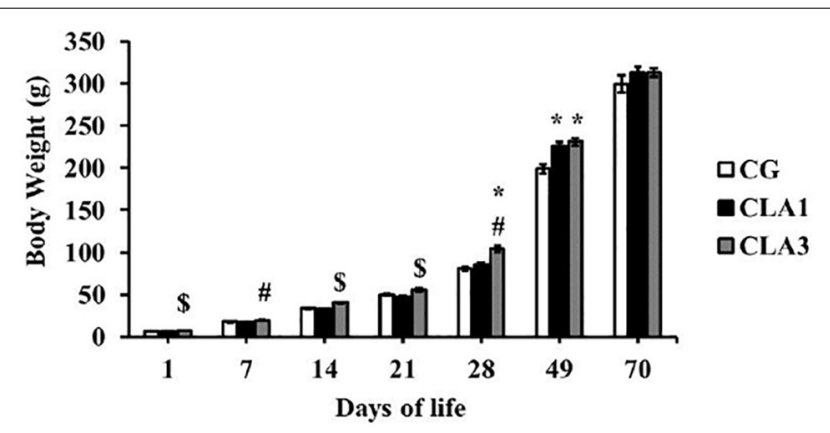

FIGURE 2 | Body weight of rats during lactation to the beginning of adulthood, treated with diet containing 1\% CLA (CLA1) or 3\% CLA (CLA3). Values are expressed as means and standard error (one way ANOVA, Holm-Sidak); * versus CG ${ }^{\#}$ versus CLA1 group; ${ }^{\$}$ versus all group. $(p<0.05)$. For the other parameters, there were no significant statistical differences.

\section{Reflex Ontogeny in Newborn Pups}

From evaluation of reflex maturation as measured in this study (Table 5), we observed that palmar grasp disappearance in the CLA1 and CLA3 groups was delayed as compared to the CG; and the CLA3 group was delayed as compared to the CLA1 group $(p<0.05)$. When maturation of cliff avoidance, vibrissa placing, negative geotaxis, and auditory startle were investigated, the CLA3 group presented acceleration as compared to the control group (CG) $(p<0.05)$. The CLA3 animals also presented increased righting and vibrissa placing in relation to the CLA1 group $(p<0.05)$. CLA1 showed acceleration of palmar grasp versus CLA3 and negative-geotaxis versus CG $(p<0.05)$. In summary, the CLA3 presented acceleration of four reflexes and CLA1 three reflexes of the seven evaluated.

\section{Open Field Habituation}

In Figure $\mathbf{3 A}$, the CLA1 and CLA3 groups spent less time ambulating in the second exposure to the open field when compared to the first exposure $(p<0.05)$. In Figure $\mathbf{3 B}$, it is possible to observe a smaller number of crossings in the fields of the open field in CLA3 in the second exposure $(p<0.05)$. The other groups did not present a statistically significant difference $(p>0.05)$. When the time spent in the internal zone was evaluated, CG spent less time in the internal zone when compared to the first and second exposures. CLA1 and CLA3 spent a longer

TABLE 4 | Evaluation of parameters after birth.

\begin{tabular}{lccc}
\hline Birth data & Control & CLA1 & CLA3 \\
\hline Litter size* $^{*}$ & $8.0 \pm 3.6$ & $13.0 \pm 0.9^{*}$ & $13.0 \pm 0.5^{*}$ \\
Number of males & $4.0 \pm 1.0$ & $7.0 \pm 2.6$ & $7.0 \pm 0.5$ \\
Number of females & $4.0 \pm 2.7$ & $6.0 \pm 1.9$ & $6.0 \pm 1.1$ \\
Birth weights $(\mathrm{g})$ & $6.0 \pm 0.6$ & $6.0 \pm 0.2$ & $7.0 \pm 0.9$ \\
\hline
\end{tabular}

* Three litters per each group.

TABLE 5 | Reflex maturation of rats which mothers were treated during gestation and lactation with standard diet containing soybean oil and other two groups with experimental diets, one containing 1\% CLA (CLA1) and the other with 3\% CLA (CLA3).

\begin{tabular}{lccc}
\hline Reflex maturation & \multicolumn{3}{c}{ Diets } \\
\cline { 2 - 4 } & $\begin{array}{c}\text { Control } \\
(\boldsymbol{n}=\mathbf{1 1})\end{array}$ & $\begin{array}{c}\text { CLA1 } \\
(\boldsymbol{n}=\mathbf{1 6})\end{array}$ & $\begin{array}{c}\text { CLA3 } \\
(\boldsymbol{n}=\mathbf{1 2})\end{array}$ \\
\hline Palmar grasp $^{\mathrm{a}}$ & $3(3-6)$ & $3.5(2-10)^{*}$ & $5(2-10)^{*} \#$ \\
Righting $^{\text {b }}$ & $3(1-6)$ & $3(2-6)$ & $2(1-4)^{\#}$ \\
Cliff-avoidance $^{\mathrm{b}}$ & $10(6-13)$ & $7.5(3-13)$ & $6(5-7)^{*}$ \\
Vibrissa-placing $^{\mathrm{b}}$ & $11(8-12)$ & $10(4-13)$ & $6(3-10)^{*} \#$ \\
Negative-geotaxis & $17(8-18)$ & $13(11-17)^{*}$ & $13(8-16)^{*}$ \\
Auditory-startle response $^{\mathrm{b}}$ & $14(12-17)$ & $13(12-15)$ & $12(12-13)^{*}$ \\
Free-fall righting $^{\mathrm{b}}$ & $11(8-15)$ & $12(9-14)$ & $13(10-16)^{*}$ \\
\hline
\end{tabular}

Values expressed as median (minimum-maximum); (one-way ANOVA, KruskalWallis); * versus Control group; " versus CLA1; a desappearance; 'bappearance. 

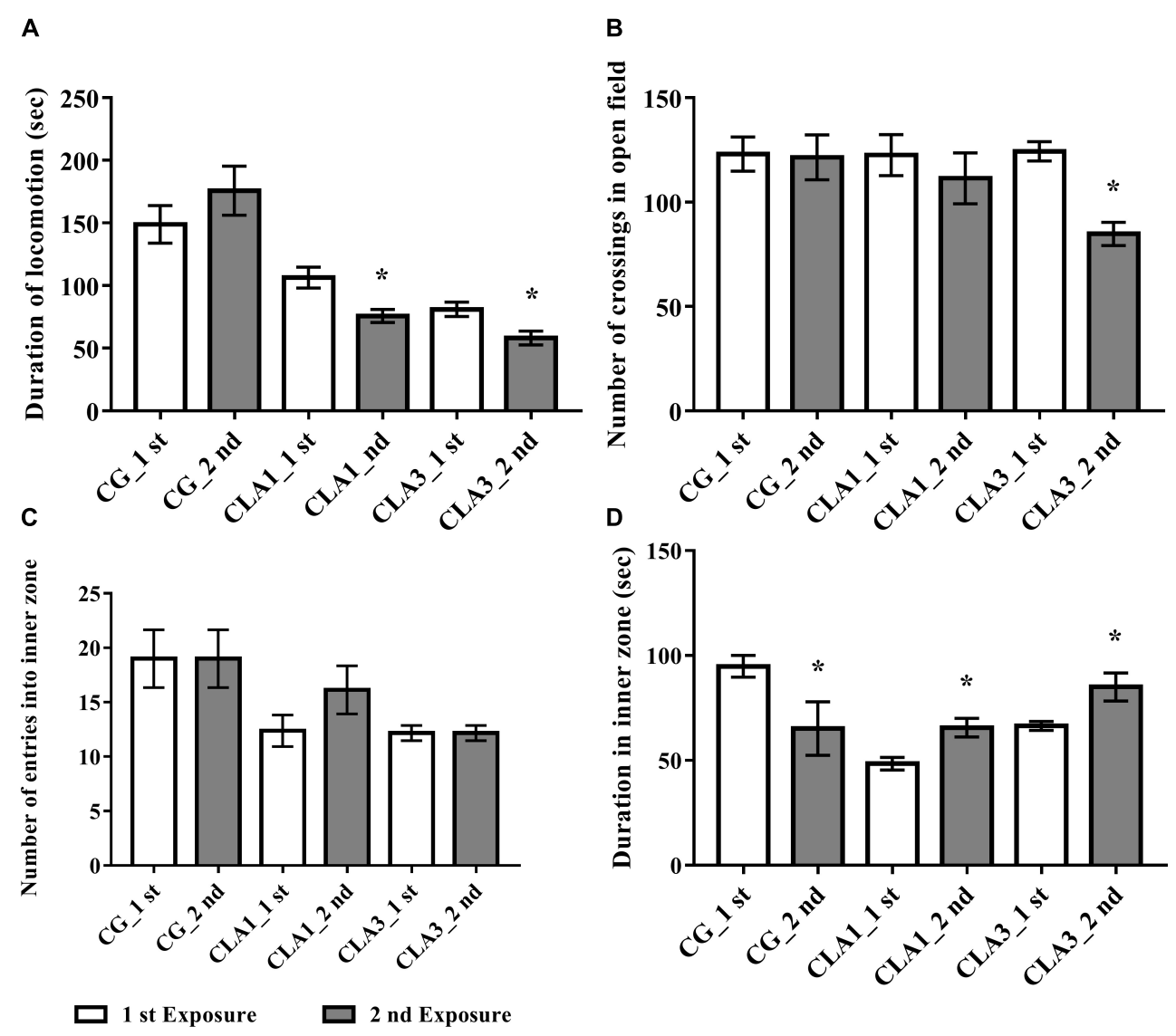

FIGURE 3 | Habituation test with newborn rats treated with standard diet (CG), with 1\% CLA (CLA1), or 3\% CLA (CLA3) during pregnancy and lactation (maternal diet). Values are expressed as means and standard deviation (one way ANOVA, Holm-Sidak); 1 st exposure to 42 days of life and 2 nd exposure to 49 days of life; CG ( $n=11)$, CLA1 $(n=13)$, CLA3 $(n=12) ;{ }^{*}$ versus first exposure in the same group. (A) Duration of locomotion: time spent by the animal moving in the open field. (B) Number of crossings in open field: the ambulation was evaluated by the total of segments covered. It was counted when the animal inserted the four legs inside the segments. (C) Number of entries into inner zone: quantified when the animal placed the four legs inside each inner zone of the open field. (D) Duration in inner zone - time spent by the animal in the inner zone of the open field.

time in the internal zone when comparing the two exposures $(p<0.05)$ (Figure 3D). The number of entry in the internal zone did not differ between the groups (Figure 3C).

\section{Object Recognition}

For short-term memory testing, there were no significant differences between the groups (Figure 4A). In the long-term memory test, all groups (CG, CLA1, and CLA3) explored unfamiliar objects more than familiar objects (Figure 4B) $(p=0.0373)$, the CLA1 group presented an increased long-term exploration ratio as compared to the CG and the CLA3 group (Figure 5B). In the short-term CLA 1 and CLA3 presented higher exploration ratio compared with CG $(p<0.05)$ (Figure 5A).

\section{Fatty Acid Profiles in Milk}

The means and standard deviations of FAs obtained from the available milk samples are presented in Table 6. The FA milk profiles from the animals (three differing diets) was in general similar, although a decrease in cis-monounsaturated fatty acid (MUFA), and an increase of total PUFA with CLA3 treatment as compared to the other treatments is suggested. The increase in total PUFA was due to the proportional increase of CLA in the milk presented by maternal (lactating) rats fed CLA supplemented diets. In fact, the control milk samples contained only trace amounts of the 18:2c9,t11 isomer, and presented no $18: 2 \mathrm{t} 10 \mathrm{c} 12$, whereas the CLA1 and CLA3 group milk samples respectively contained about 1 and $3 \%$ of total FA for each CLA isomer.

\section{Fatty Acids and Dimethyl Acetal in the Brain}

The fatty acid and DMA profiles in the brain tissue of the pups are presented in Table 7 . The treatments significantly affected most of the FA and DMA profiles, although they did not follow the expected response patterns. In fact, most of the FA and DMA profiles differed between the controls and the CLA1 pups, whereas the CLA3 pups were either similar to the controls (14:0, 23:0, 16:1c7, 18:1c9, 18:1c11, 20:1c11, 24:1c15, 18:2n-6, 20:2n-6, 20:3n-6, cis-MUFA, n-6 PUFA, total PUFA, DMA-17:0, DMA18:1c9, DMA-18:1c11, and total DMA), or between the controls 

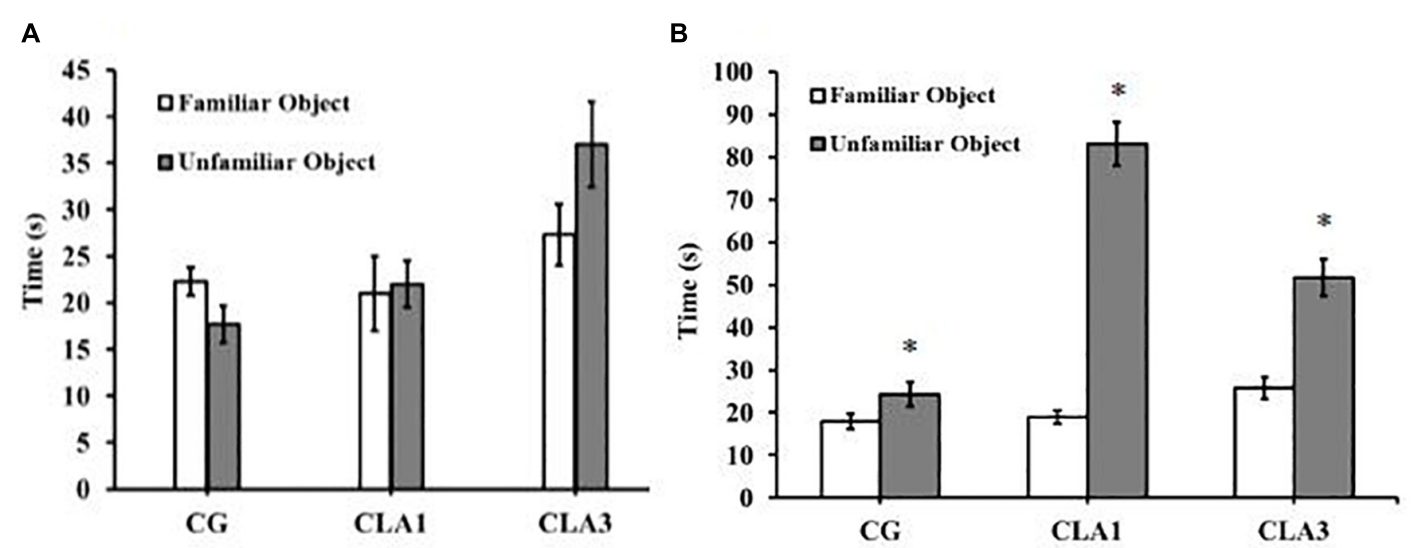

FIGURE 4 | Object recognition test in rats treated with 1\% (CLA1) or 3\% CLA (CLA3) during pregnancy and lactation (maternal diet). CG: Control group without CLA in the diet. Values are expressed as means and standard deviation (one way ANOVA, Holm-Sidak). (A) Short-term test using familiar object (A1) and unfamiliar object (A3). (B) Long-term test using familiar object (A1) and unfamiliar object (A4); CG ( $n=11)$, CLA1 ( $n=13)$, CLA3 $(n=12)$; * versus familiar object in the same group.
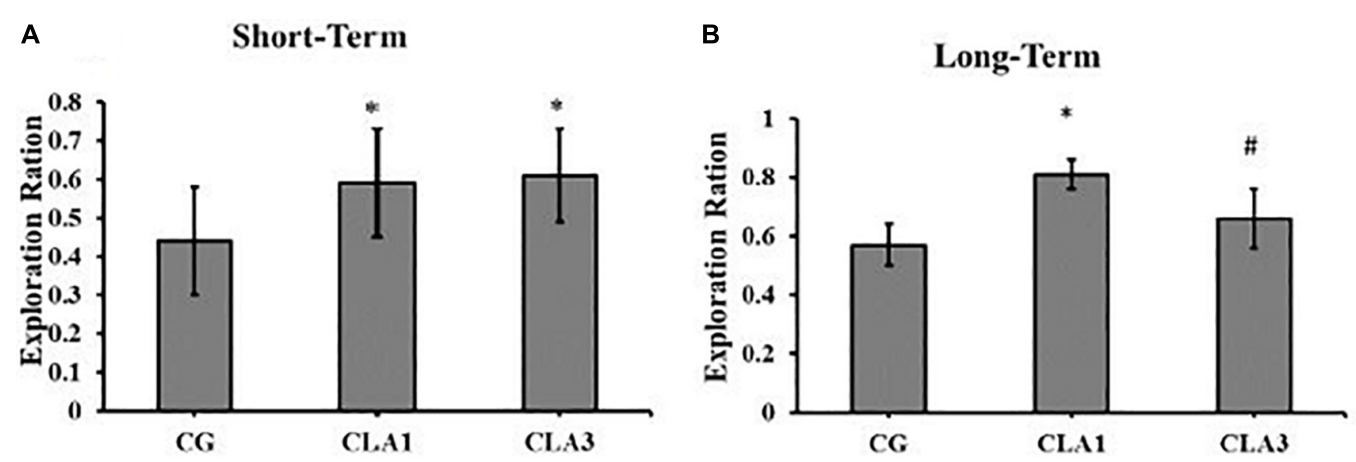

FIGURE 5 | Exploration Ratio for object recognition test in rats treated during pregnancy and lactation (maternal diet) with 1\% (CLA1) or 3\% CLA (CLA3). CG: Control group without CLA in the maternal diet. Values are expressed as means and standard deviation (one way ANOVA, Holm-Sidak). (A) Short-term test using familiar object (A1) and unfamiliar object (A3). (B) Long-term test using familiar object (A1) and unfamiliar object (A4); CG ( $n=11)$, CLA1 ( $n=13)$, CLA3 ( $n=12$ );

* versus CG; \# versus CLA1 group.

and the CLA1 pups (16:0, 20:0, 24:0, 16:1c9, 20:1, 22:1c13, 24:1, 20:4n-6, 22:4n-6, 22:5n-6, 22:6n-3, SFA, n-3 PUFA, and DMA18:0). Thus, the brains of the CLA1 pups presented higher $(p<0.05)$ proportions of SFA and PUFA, but lower $(p<0.05)$ proportions of MUFA and total DMA than the controls. The CLA isomers were not detected in the brains of the CG pups, and were present only as trace amounts in the CLA1 and CLA3 pups.

\section{DISCUSSION}

This study evaluated the developmental effects of maternal supplementation with differing CLA concentrations (1 and $3 \%$ ) administered during gestation and lactation in their offspring. The data presented the significant effects of CLA on physical growth (with increases in body weight), and on neurodevelopment (acceleration in reflex maturation and memory improvement) in the newborn rats.

Neurodevelopment occurs in the perinatal period, and lipids are increasingly recognized as playing an important role in neuronal function in the brain (Morgane et al., 1993; Salvati et al., 2000). During this phase, essential fatty acids of the n-3 and n6 families together constitute a lipid substrate that is required for adequate formation of nerve cell membranes. The lipids are also involved in cell signaling and regulate synaptic throughput (Muller et al., 2015). The effects of n-3 in the maternal diet on brain development of the offspring are already known (Rathod et al., 2014; Mucci et al., 2015), However, the effect of other fatty acids like CLA have not yet been investigated. In this study, a maternal diet that included CLA, induced improvements in memory, and accelerated neurodevelopment in the offspring.

Maternal diets containing different $n-6 / n-3$ ratios in rats have been associated with reflex ontogeny and physical growth modifications in the offspring (Santillan et al., 2010; Aquino et al., 2015). Such results demonstrate early life vulnerability of developing nervous systems to an inadequate balance of essential fatty acids. Treatment of Wistar rats during lactation with goat milk (fat containing CLA) has been shown to result in cliff avoidance delays, but there were anticipations in free-fall righting (Soares et al., 2013). Another study (Santillan et al., 2010) found 
TABLE 6 | Fatty acid composition (means \pm standard deviation expressed as $\mathrm{g} / \mathrm{kg}$ of total fatty acids) of breast milk of dams fed control, CLA1 and CLA2 diets.

\begin{tabular}{|c|c|c|c|}
\hline Fatty acids & Control & CLA1 & CLA3 \\
\hline$n^{1}$ & 1 & 3 & 2 \\
\hline $8: 0$ & 29.5 & $37.9 \pm 11.1$ & $52.5 \pm 3.07$ \\
\hline $10: 0$ & 92.7 & $104 \pm 42.9$ & $148 \pm 15.9$ \\
\hline $12: 0$ & 61.9 & $56.0 \pm 27.3$ & $80.1 \pm 14.2$ \\
\hline $14: 0$ & 54.3 & $41.3 \pm 15.7$ & $49.9 \pm 10.4$ \\
\hline 15:0 & 1.05 & $1.24 \pm 0.18$ & $1.12 \pm 0.29$ \\
\hline $16: 0$ & 219 & $201 \pm 24.0$ & $159 \pm 3.3$ \\
\hline $17: 0$ & 0.98 & $1.16 \pm 0.20$ & $1.03 \pm 0.19$ \\
\hline 18:0 & 31.0 & $36.4 \pm 4.44$ & $31.5 \pm 1.77$ \\
\hline $20: 0$ & 0.72 & $0.80 \pm 0.07$ & $0.95 \pm 0.04$ \\
\hline 22:0 & 0.20 & $0.38 \pm 0.04$ & $0.28 \pm 0.02$ \\
\hline $23: 0$ & 0.23 & $0.29 \pm 0.05$ & $0.31 \pm 0.10$ \\
\hline $24: 0$ & 0.36 & $0.40 \pm 0.04$ & $0.51 \pm 0.06$ \\
\hline $\mathrm{SFA}^{2}$ & 492 & $481 \pm 68.6$ & $524 \pm 44.6$ \\
\hline $14: 1 c 9$ & 1.00 & $0.74 \pm 0.29$ & $0.34 \pm 0.12$ \\
\hline $16: 1 c 7$ & 3.25 & $3.05 \pm 0.90$ & $1.99 \pm 0.39$ \\
\hline $16: 1 c 9$ & 36.4 & $26.6 \pm 9.27$ & $12.0 \pm 1.50$ \\
\hline $17: 1 c 9$ & 1.16 & $1.09 \pm 0.29$ & $0.68 \pm 0.14$ \\
\hline $18: 1 t 6 / t 7 / t 8$ & 0.17 & $0.29 \pm 0.02$ & $0.25 \pm 0.02$ \\
\hline $18: 1 t 9$ & 0.22 & $0.27 \pm 0.08$ & $0.23 \pm 0.01$ \\
\hline $18: 1 t 10$ & 0.17 & $0.35 \pm 0.09$ & $0.56 \pm 0.16$ \\
\hline $18: 1 t 11$ & 0.53 & $0.68 \pm 0.01$ & $1.01 \pm 0.44$ \\
\hline $18: 1 t 12$ & 0.24 & $0.41 \pm 0.06$ & $0.34 \pm 0.01$ \\
\hline $18: 1 c 9$ & 230 & $222 \pm 48.1$ & $159 \pm 19.5$ \\
\hline $18: 1 c 11$ & 17.6 & $18.1 \pm 4.40$ & $11.7 \pm 1.89$ \\
\hline $18: 1 c 13$ & 0.46 & $0.52 \pm 0.03$ & $0.42 \pm 0.18$ \\
\hline $24: 1 c 15$ & 0.10 & $0.23 \pm 0.10$ & $0.11 \pm 0.004$ \\
\hline cis-MUFA ${ }^{3}$ & 290 & $273 \pm 61.3$ & $186 \pm 20.6$ \\
\hline trans-MUFA ${ }^{4}$ & 1.34 & $2.27 \pm 019$ & $3.05 \pm 0.61$ \\
\hline $18: 2 n-6$ & 187 & $193 \pm 15.3$ & $187 \pm 18.1$ \\
\hline $18: 3 n-6$ & 1.83 & $1.41 \pm 0.76$ & $0.94 \pm 0.08$ \\
\hline $20: 2 n-6$ & 2.67 & $3.43 \pm 0.47$ & $3.14 \pm 0.30$ \\
\hline $20: 3 n-6$ & 1.59 & $1.63 \pm 0.58$ & $1.06 \pm 0.05$ \\
\hline $20: 4 n-6$ & 5.35 & $6.52 \pm 1.70$ & $6.26 \pm 0.64$ \\
\hline n-6 PUFA 5 & 199 & $206 \pm 18.1$ & $198 \pm 19.0$ \\
\hline $18: 3 n-3$ & 13.0 & $14.7 \pm 2.47$ & $16.4 \pm 0.78$ \\
\hline $20: 3 n-3$ & 0.43 & $0.47 \pm 0.06$ & $0.50 \pm 0.03$ \\
\hline $20: 5 n-3$ & 0.38 & $0.42 \pm 0.08$ & $0.49 \pm 0.01$ \\
\hline $22: 5 n-3$ & 0.63 & $0.70 \pm 0.09$ & $0.66 \pm 0.09$ \\
\hline $22: 6 n-3$ & 0.78 & $1.06 \pm 0.43$ & $1.24 \pm 0.01$ \\
\hline n-3 PUFA ${ }^{6}$ & 15.2 & $17.3 \pm 2.77$ & $19.3 \pm 0.69$ \\
\hline $18: 2 c 9 t 11$ & 0.42 & $10.6 \pm 1.84$ & $35.0 \pm 1.86$ \\
\hline $18: 2 t 10 c 12$ & 0.00 & $7.8 \pm 1.85$ & $29.6 \pm 1.03$ \\
\hline $18: 2 c 9 c 11$ & 0.19 & $0.53 \pm 0.09$ & $0.91 \pm 0.13$ \\
\hline Other CLA & 0.50 & $1.17 \pm 0.06$ & $2.22 \pm 0.44$ \\
\hline Total CLA & 1.11 & $20.1 \pm 3.73$ & $67.8 \pm 3.47$ \\
\hline Total PUFA & 216 & $244 \pm 18.2$ & $286 \pm 23.4$ \\
\hline
\end{tabular}

${ }^{1}$ Number of samples; ${ }^{2}$ Sum of saturated fatty acids; ${ }^{3}$ Sum of cis monounsaturated fatty acids; ${ }^{4}$ Sum of trans monounsaturated fatty acids; ${ }^{5}$ Sum of $n-6$ polyunsaturated fatty acids; ${ }^{6}$ Sum of $n-3$ polyunsaturated fatty acids.

a delay for negative geotaxis in animals consuming soybean oil, yet an acceleration for cliff avoidance in animals treated with sunflower oil. The cerebellum presents peak development during lactation, and reflex maturity is directly related to the continuous differentiation and maturation of cerebellar neurons (Allam and Albo-Eleneen, 2012), which involve the visual and postural systems (Boyle, 2001).

In the present study, 1\% CLA induced acceleration for palmar gasp and negative geotaxis, however when the dose was tripled, cliff-avoidance, vibrissa-placing, negative-geotaxis, auditory-startle response, were accelerated. The group treated with CLA3\% obtained a better response in four parameters of the seven evaluated for reflex. On the other hand, the CLA1 group presented two accelerated reflexes. When analyzing the two groups it can be stated that the effects were moderate. It is unclear what mechanisms the body uses to transform CLA into PUFAs, what is known is that no large doses of omega-3 lipids are required for a better response in the nervous system. In addition, high doses of this lipid may cause depletion of omega- 6 lipids and the consequent imbalance between n-3 and n-6 (Zanarini and Frankenburg, 2003; Salvati et al., 2006). These findings suggest that CLA positively affects neurodevelopment, anticipating reflex maturation in the offspring.

Studies show that CLA can pass through the blood-brain barrier (Fa et al., 2005; Soares et al., 2012), reaching the brain, where it performs beneficial functions. However in our study, CLA was found in the brain only in trace amounts. In the milk, in the CLA1 and CLA3 groups, apparently the quantities of CLA were higher, but this cannot be stated with accuracy since statistical analysis was not performed, since some of the samples were lost during transit. This may be considered a limitation of our study.

Thus, we believe that in the present study the effects of this fatty acid occurred indirectly. Study analyzed aspects of maternal metabolism on milk composition show that the transfer of fatty acids to the mother's milk may vary depending on their quality as consumed in the maternal diet (Demmelmair and Koletzko, 2018). The authors observed that alpha-linolenic acid offered in the diet contributes more than that of the normal maternal reserve to its final quantity as found in the milk. However, linoleic acid in breast milk is derived more from the liver than from the diet itself (Demmelmair and Koletzko, 2018). The literature reports on metabolism and omega 3 and 6 in breast milk (Demmelmair and Koletzko, 2018). However, little is known about the influence of maternal consumption of CLA on the composition of breast milk.

In rats fed a diet rich in trans-FAs, offered during early life and after weaning, spatial memory was modified (Souza et al., 2012), and a maternal diet containing high levels of lard and saturated fats was found to induce damages to the memory and learning ability of the offspring (Yu et al., 2010). However, both studies cited, evaluated memory using the Morris water maze test. In our study, new object recognition test was used to assess working memory. Both groups treated with CLA (CLA1 and CLA3) demonstrated increased time of exploration of unfamiliar (novel) objects, indicating a better working memory. This findings differ from the results reported by Yu et al. (2010), who showed that offsprings of mice treated with saturated fats performed worse in Morris water maze test. We should point 
TABLE 7 | Fatty acids (FAs) and dimethyl acetals (DMAs) composition (least square means \pm standard error, expressed as $\mathrm{g} / \mathrm{kg}$ of total FA + DMA) cerebral tissue of offspring from dams fed control, CLA1 and CLA3 diets.

\begin{tabular}{|c|c|c|c|c|}
\hline & Control & CLA1 & CLA3 & $P$-value \\
\hline$n^{1}$ & 6 & 4 & 4 & \\
\hline \multicolumn{5}{|l|}{ FA } \\
\hline $14: 0$ & $1.13 \pm 0.07$ & $3.54 \pm 0.21^{*}$ & $1.77 \pm 0.43^{\#}$ & $<0.001$ \\
\hline $15: 0$ & $0.38 \pm 0.03$ & $0.63 \pm 0.06^{*}$ & $0.52 \pm 0.02 *$ & 0.003 \\
\hline $16: 0$ & $179 \pm 0.8$ & $216 \pm 4.0^{*}$ & $197 \pm 10.8$ & $<0.001$ \\
\hline $17: 0$ & $1.52 \pm 0.04$ & $1.40 \pm 0.08$ & $1.65 \pm 0.17$ & 0.289 \\
\hline $18: 0$ & $207 \pm 0.9$ & $212 \pm 1.92^{*}$ & $211 \pm 1.5$ & 0.025 \\
\hline $20: 0$ & $5.86 \pm 0.08$ & $4.25 \pm 0.35^{*}$ & $5.02 \pm 0.54$ & 0.003 \\
\hline $22: 0$ & $5.87 \pm 0.12$ & $4.93 \pm 0.41$ & $5.08 \pm 0.52$ & 0.075 \\
\hline $23: 0$ & $1.60 \pm 0.07$ & $0.45 \pm 0.09^{*}$ & $1.23 \pm 0.33^{\#}$ & $<0.001$ \\
\hline $24: 0$ & $12.3 \pm 0.16$ & $8.39 \pm 0.87^{*}$ & $9.75 \pm 1.41$ & 0.002 \\
\hline $\mathrm{SFA}^{2}$ & $414 \pm 0.6$ & $452 \pm 3.7^{*}$ & $433 \pm 9.7$ & $<0.001$ \\
\hline $16: 1 c 7$ & $1.21 \pm 0.06$ & $3.12 \pm 0.11^{*}$ & $1.82 \pm 0.44$ & $<0.001$ \\
\hline $16: 1 c 9$ & $3.12 \pm 0.18$ & $4.06 \pm 0.01^{*}$ & $3.55 \pm 0.28$ & 0.001 \\
\hline $17: 1 c 9$ & $0.36 \pm 0.04$ & $0.27 \pm 0.05$ & $0.44 \pm 0.08$ & 0.209 \\
\hline 18:1trans & $2.18 \pm 0.16$ & $2.75 \pm 0.16$ & $2.23 \pm 0.29$ & 0.066 \\
\hline $18: 1 c 9$ & $154 \pm 0.6$ & $122 \pm 3.1^{*}$ & $141 \pm 7.9^{\#}$ & $<0.001$ \\
\hline $18: 1 c 11$ & $29.7 \pm 0.17$ & $24.9 \pm 0.61^{*}$ & $28.7 \pm 0.93^{\#}$ & $<0.001$ \\
\hline $20: 1 c 11^{3}$ & $12.9 \pm 0.21$ & $5.46 \pm 0.49^{*}$ & $9.79 \pm 1.87^{\#}$ & $<0.001$ \\
\hline $20: 1^{4}$ & $4.03 \pm 0.05$ & $1.77 \pm 0.19^{*}$ & $3.08 \pm 0.58$ & $<0.001$ \\
\hline $22: 1 c 13$ & $1.85 \pm 0.05$ & $0.95 \pm 0.08^{*}$ & $1.46 \pm 0.28$ & 0.012 \\
\hline $24: 1 c 15$ & $18.1 \pm 0.46$ & $7.41 \pm 0.84^{*}$ & $14.3 \pm 2.96^{\#}$ & $<0.001$ \\
\hline $24: 1^{4}$ & $1.92 \pm 0.05$ & $0.94 \pm 0.09^{*}$ & $1.58 \pm 0.32$ & $<0.001$ \\
\hline cis-MUFA ${ }^{5}$ & $230 \pm 0.8$ & $173 \pm 5.4^{*}$ & $208 \pm 14.1^{\#}$ & $<0.001$ \\
\hline $18: 2 n-6$ & $6.12 \pm 0.06$ & $12.1 \pm 0.32 *$ & $7.00 \pm 1.25^{\#}$ & $<0.001$ \\
\hline $20: 2 n-6$ & $1.32 \pm 0.07$ & $2.58 \pm 0.09^{*}$ & $1.50 \pm 0.20^{\#}$ & $<0.001$ \\
\hline $20: 3 n-6$ & $3.51 \pm 0.08$ & $5.97 \pm 0.34^{*}$ & $3.65 \pm 0.26^{\#}$ & $<0.001$ \\
\hline $20: 4 n-6$ & $98 \pm 0.65$ & $115 \pm 2.12^{*}$ & $104 \pm 4.47$ & $<0.001$ \\
\hline $22: 4 n-6$ & $29.1 \pm 0.27$ & $30.9 \pm 0.52$ & $29.7 \pm 0.55$ & 0.029 \\
\hline $22: 5 n-6$ & $7.05 \pm 0.21$ & $9.13 \pm 0.27^{*}$ & $7.68 \pm 0.87$ & $<0.001$ \\
\hline$n-6$ PUFA $^{6}$ & $145 \pm 1.0$ & $176 \pm 2.7^{*}$ & $154 \pm 7.4^{\#}$ & $<0.001$ \\
\hline $22: 5 n-3$ & $2.33 \pm 0.04$ & $3.19 \pm 0.18^{*}$ & $2.14 \pm 0.05^{* \#}$ & $<0.001$ \\
\hline $22: 6 n-3$ & $116 \pm 0.9$ & $123 \pm 1.9 *$ & $120 \pm 3.2$ & 0.017 \\
\hline n-3 PUFA ${ }^{7}$ & $118 \pm 0.9$ & $127 \pm 1.8$ & $122 \pm 3.1$ & 0.030 \\
\hline $20: 2^{4}$ & $1.09 \pm 0.05$ & $0.79 \pm 0.06^{*}$ & $0.86 \pm 0.03^{*}$ & 0.005 \\
\hline $20: 3 n-9$ & $0.82 \pm 0.06$ & $0.69 \pm 0.02$ & $0.66 \pm 0.03$ & 0.101 \\
\hline $18: 2 c 9 t 11$ & nd & $0.08 \pm 0.06$ & $0.24 \pm 0.08$ & 0.154 \\
\hline $18: 2 t 10 c 12$ & nd & $0.05 \pm 0.04$ & $0.15 \pm 0.05$ & 0.171 \\
\hline Total CLA & nd & $0.13 \pm 0.09$ & $0.39 \pm 0.13$ & 0.159 \\
\hline Total PUFA & $266 \pm 0.9$ & $304 \pm 3.9^{*}$ & $277 \pm 10.3^{\#}$ & $<0.001$ \\
\hline \multicolumn{5}{|l|}{ DMA } \\
\hline $16: 0$ & $20.6 \pm 0.35$ & $23.8 \pm 0.27^{*}$ & $22.1 \pm 0.27^{* \#}$ & $<0.001$ \\
\hline $17: 0$ & $0.85 \pm 0.05$ & $0.45 \pm 0.06^{*}$ & $0.84 \pm 0.16^{\#}$ & $<0.001$ \\
\hline 18:0 & $42.3 \pm 0.36$ & $33.4 \pm 0.62^{*}$ & $37.7 \pm 2.69$ & $<0.001$ \\
\hline $18: 1 c 9$ & $15.4 \pm 0.15$ & $8.1 \pm 0.57^{*}$ & $12.4 \pm 1.95^{\#}$ & $<0.001$ \\
\hline $18: 1 c 11$ & $11.5 \pm 0.18$ & $5.01 \pm 0.29^{*}$ & $9.14 \pm 1.72^{\#}$ & $<0.001$ \\
\hline Total DMA & $90.6 \pm 0.66$ & $70.8 \pm 1.44^{*}$ & $82.3 \pm 6.20^{\#}$ & $<0.001$ \\
\hline
\end{tabular}

${ }^{1}$ number of samples; ${ }^{2}$ Sum of saturated fatty acids; ${ }^{3}$ Coelutes with minor amounts of 18:3n-3; ${ }^{4}$ Double bond position not determined; ${ }^{5}$ Sum of cis-monounsaturated fatty acids; ${ }^{6}$ Sum of $n-6$ polyunsaturated fatty acids; ${ }^{7}$ Sum of $n-3$ polyunsaturated fatty acids; * versus CG ${ }^{\#}$ versus CLA1 group. out that we did different memory test and CLA isomers are unsaturated fat.

In early stage of life in the rat, neurodevelopment happens very fast, as demonstrated when was measured the reflex maturation in the present study. The reflex maturation occurred from birth to 18 days of life. In addition, maternal supplementation with CLA altered the deposition of fatty acids in the brain as demonstrated in Table 7. Unfortunately, we evaluated the composition of fatty acids in this tissue only at the end of the experiment (70 days of life), which made it impossible to evaluate the transition of fatty acids in this brain tissue and to relate to the transitional period that led to the consolidation of the memory by the animals. However, the exploration index by CLA groups were always higher than CG (short and long time). Thus, these data confirm how neurodevelopment occurs fast in the nervous system, even during lactation with the evaluation of reflex maturation and in the postnatal period with evaluated of memory by the object recognition test.

The experimental diets significantly affected most of the fatty acids and DMA, although they did not follow the expected response patterns. The CLA1 group presented higher amounts of PUFAs as compared to the CG, and the CLA3 group's PUFA levels were intermediates between those of the CLA1 and CG groups. Among the polyunsaturated fats found in the brain for the CLA1 group we detected AA and DHA. In the brain, DHA (22:6n-3) and AA (20:4n-6) have been correlated with better spatial memory performance (Fernandes et al., 2011; Harauma et al., 2017).

As was seen in the results, CLA is present in breast milk, and it may be affirmed that the pups received CLA by breastfeeding. However, in the brain, the lipid was found only in small amounts. Yet maternal consumption of CLA induced memory improvement in their offspring. Although CLA was not deposited in large amounts in the brain, its consumption significantly increased n-3 and n-6 fatty acids in the rat brain. Among these fatty acids is AA, an n-6 fatty acid that plays an important role in brain functions, including neuronal signal transmission and long-term potentiation. In addition, AA preserves hippocampal neuron membrane plasticity, protects the brain against oxidative stress, improves memory, and helps in the synthesis of new proteins in brain tissues (Hadley et al., 2016).

AA can be synthesized from substrates such as CLA and LA (Banni, 2002). These fatty acids likely share the same enzymes (desaturases and elongases). Thus, increased CLA ingestion may impede metabolism of linoleic acid, and consequently proinflammatory metabolites (Białek et al., 2015).

The principally known metabolites of CLA belong to conjugated diene (CD) structured compounds, such as conjugated octadecetrienoic acid (CD18: 3), conjugated eicosatrienoic acid (CD 20:3), and conjugated eicosatetraenoic acid (CD 20: 4) which are synthesized in the desaturase and elongase pathways (Banni, 2002). However, CLA metabolism may interfere with the formation of eicosanoids, and CLA hydroxylation and its metabolites conjugated in LOX pathways, or cytochrome P450 may form eicosanoid-like molecules that 
compete with regular eicosanoids (Banni, 2002), thus potentially exerting anti-inflammatory action in many, including brain tissue (Shen et al., 2018). Further studies would be needed to elucidate the mechanism by which CLA can induce increased DHA.

Polyunsaturated fatty acids are essential for brain development and memory because they modulate synaptic plasticity, and thus improve learning ability. In human infants (our study investigates this same stage of life), accumulation of these fatty acids occurs during gestation and lactation, through the placenta and breast milk (Banni et al., 1996).

Another memory index used was the open field habituation test, and lower locomotor activity in a repeated exposure indicates good recognition (Rachetti et al., 2013). In the present study CLA1 and CLA3 presented less locomotion and spent longer time in the internal area in the second exposure into the open field. This behavior indicates that the animals moved less because they remembered the place and lost interest in exploration. The time spent in the central area confirms that this decrease in locomotion in the second moment did not occur due to the behavior like anxiogenic of the animals.

A diet enriched in n-3 PUFA has induced an increase in exploratory activity for young rats, which was not observed in the mature or older rats tested (Das, 2003). However, a maternal CLA enriched diet reduced ambulatory activity, as demonstrated in the present study. Thus, it is demonstrated for the first time that CLA is able to affect these learning parameters. With greater CLA levels in the diet, levels of CLA isomers (18:2 cis-9, trans-11 and 18:2 trans-10, cis-12) increase in the milk. Yet in the brain, these CLA isomers were present only in trace amounts; and although proportionally higher in the CLA3 group as compared to the CLA1 group, the difference was not significant.

Maternal dietary lipids may also affect body weight in offspring. In this study, the body weight of pups whose mothers received 3\% CLA was higher throughout and after lactation. A similar result was observed when during gestation and lactation (Soares et al., 2012) rats received a diet containing goat milk (which is a source of CLA). A different result was observed when lactating rats received 1.35\% CLA (Hayashi et al., 2007). Maternal treatment during pregnancy and lactation with $1.47 \%$ CLA resulted in pups presenting decreased body weights (Ringseis et al., 2004). The authors attribute this effect to lipid reduction in the breast milk; and yet such a reduction was not induced in the present study. The data demonstrate that maternal dietary lipids may differently affect the physical parameters of their offspring. Evaluating the body weight and feed intake of the progenitors, there was no statistical difference between the groups. The mothers who had the analyzed milk are representatives of their groups when evaluated body weight and feed intake.

The animals from the groups experimental presented anticipations of certain reflexes, as well as improvements in

\section{REFERENCES}

Allam, A. A., and Albo-Eleneen, R. E. (2012). The development of sensorimotor reflexes in albino mice; albino rats and black-hooded rats. Int. J. Dev. Neurosci. 30, 545-553. doi: 10.1016/j.ijdevneu.2012.08.005 memory. These findings are important because they demonstrate the benefits that CLA consumption can bring to the developing brain. The present research will serve to guarantee a safety indication of the consumption of CLA by pregnant and lactating women by doctors and nutritionists. In addition, the supplement used in this research is a trademark and can be freely purchased by individuals and verify the effects of its consumption is very important. However, it is interesting to conduct research with humans to guarantee the similarity of the results found in the present research with women and infants.

\section{CONCLUSION}

Based on our results, it may be concluded that maternal supplementation with CLA influenced development of the offspring central nervous system, accelerating reflex maturation (Cliff-avoidance, Vibrissa-placing, Negative-geotaxis, Auditorystartle response) and delay in only two reflexes (Palmar grasp and Free-fall righting) in the group which received 3\% CLA. In the group which received 1\% CLA was observed acceleration in reflexes (Palmar grasp and Negative-geotaxis). Memory improvement was also observed in CLA treated groups where there was greater exploration of the new object in the object recognition test. It is suggested that further studies be performed to prove the effects of CLA on the central nervous system.

\section{ETHICS STATEMENT}

The research followed an experimental protocol in accordance with the ethical recommendations of the National Institutes of Health (Bethesda, MD, United States), and was approved by the ethics research committee of the Federal University of Paraíba No. 0407/13.

\section{AUTHOR CONTRIBUTIONS}

JS, CB, MM, MO, and RQ designed the theme of the study. MB and $\mathrm{ML}$ performed the designed experimental methods. RB, SA, and MS for conducting the analysis of fatty acids. JS, MM, MB, and MQ analyzed the data. JS and MQ interpreted the results and wrote the article. This research was carried out by all authors.

\section{FUNDING}

This study was financed in part by the Coordenação de Aperfeiçoamento de Pessoal de Nível Superior - Brasil (CAPES) Finance Code 001.

Alves, S. P., Santos-Silva, J., Cabrita, A. R. J., Fonseca, A. J. M., and Bessa, R. J. B. (2013). Detailed dimethylacetal and fatty acid composition of rumen content from lambs fed lucerne or concentrate supplemented with soybean oil. PLoS One 8:e58386. doi: 10.1371/journal.pone.005 8386 
Aquino, J. S., Soares, J. K. B., Magnani, M., Stamford, T. C. M., Mascarenhas, R. J., Tavares, R. L., et al. (2015). Effects of dietary brazilian palm oil (Mauritia flexuosa L.) on cholesterol profile and vitamin A and E status of rats. Molecules 20, 9054-9070. doi: 10.3390/molecules20059054

Balogun, K. A., Randunu, R. S., and Cheema, S. K. (2014). The effect of dietary omega-3 polyunsaturated fatty acids on plasma lipids and lipoproteins of C57BL/6 mice is age and sex specific. Prost. Leuk. Essen. Fat. Acids 91, 39-47. doi: 10.1016/j.plefa.2014.05.002

Banni, S. (2002). Conjugated linoleic acid metabolism. Curr. Opin. Lipidol. 13, 261-266. doi: 10.1097/00041433-200206000-00005

Banni, S., Carta, G., Contini, M. S., Angioni, E., Deiana, M., Dessi, M. A., et al. (1996). Characterization of conjugated diene fatty acids in milk, dairy products, and lamb tissues. J. Nutr. Biochem. 7, 150-151. doi: 10.1016/0955-2863(95) 00193-X

Białek, A., Jelińska, M., and Tokarz, A. (2015). Influence of maternal diet enrichment with conjugated linoleic acid on lipoxygenase metabolites of polyunsaturated fatty acids in serum of their offspring with 7,12dimethylbenz[a]anthracene induced mammary tumors. Prost. Other Lipid Med. 11, 10-18. doi: 10.1016/j.prostaglandins.2014.10.001

Boyle, R. (2001). Vestibulospinal control of reflex and voluntary head movement. Ann. N. Y. Acad. Sci. 942, 364-380. doi: 10.1111/j.1749-6632.2001.tb0 3760.x

Chalon, S., Delion-Vascassel, S., Belzung, C., Guilloteau, D., Lequisquet, A. M., Besnard, J. C., et al. (1998). Dietary fish oil affects monoaminergic neurotransmission and behavior in rats. J. Nutr. 128, 2512-2519. doi: 10.1093/ jn/128.12.2512

Das, U. N. (2003). Can memory be improved? A discussion on the role ofras, GABA acetylcholine, NO, insulin, TNF- $\alpha$, and long-chain polyunsaturated fatty acids in memory formation and consolidation. Brain Dev. 25, 251-261. doi: 10.1016/s0387-7604(02)00221-8

Demmelmair, H., and Koletzko, B. (2018). Lipids in human milk. Best Pract. Res. Clin. Endocrinol. Metab. 32, 57-68. doi: 10.1016/j.beem.2017.11.002

Fa, M., Diana, A., Carta, G., Cordeddu, L., Melis, M. P., Murru, E., et al. (2005). Incorporation and metabolism of $\mathrm{c} 9, \mathrm{t} 11$ and $\mathrm{t} 10, \mathrm{c} 12$ conjugated linoleic acid (CLA) isomers in rat brain. Biochim. Biophys. Acta 1736, 61-66. doi: 10.1016/j. bbalip.2005.06.010

Fernandes, F. S., Souza, A. S., Carmo, M. G. T., and Boaventura, G. T. (2011). Maternal intake of flaxseed-based diet (Linum usitatissimum) on hippocampus fatty acid profile: implications for growth, locomotor activity and spatial memory. Nutrition 27, 1040-1047. doi: 10.1016/j.nut.2010.11.001

Furlan, C. P. B., Marques, A. C., Marineli, R. S., and Júnior, M. R. M. (2013). Conjugated linoleic acid and phytosterols counteract obesity induced by high-fat diet. Food Res. Int. 51, 429-435. doi: 10.1016/j.foodres.2012. 12.023

Gamberini, M. T., Rodrigues, D. S., Rodrigues, D., and Pontes, V. B. (2015). Effects of the aqueous extract of Pimpinella anisum L. seeds on exploratory activity and emotional behavior in rats using the open field and elevated plus maze tests. J. Ethnopharmacol. 8, 45-49. doi: 10.1016/j.jep.2015.03.053

Gaoa, J., Wua, H., Cao, Y., Liang, S., Sun, C., Wang, P., et al. (2016). Maternal DHA supplementation protects rat offspring against impairment of learning and memory following prenatal exposure to valproic acid. J. Nutr. Biochem. 35, 87-95. doi: 10.1016/j.jnutbio.2016.07.003

Gustavsson, M., Hodgkinson, S. C., Fong, B., Norris, C., Guan, J., Krageloh, C. U., et al. (2010). Maternal supplementation with a complex milk lipid mixture during pregnancy and lactation alters neonatal brain lipid composition but lacks effect on cognitive function in rats. Nutr. Res. 30, 279-289. doi: 10.1016/j. nutres.2010.04.005

Hadley, K. B., Ryan, A. S., Forsyth, S., Gautier, S., and Salem, N. (2016). The essentiality of arachidonic acid in infant development. Nutrients 8:216. doi: $10.3390 /$ nu8040216

Halade, G. V., Rahman, M. D. M., and Fernandes, G. (2010). Differential effects of conjugated linoleic acid isomers in insulin-resistant female C57Bl/6J mice. J. Nutr. Biochem. 21, 332-337. doi: 10.1016/j.jnutbio.2009.01.006

Harauma, A., Hatanaka, E., Yasuda, H., Nakamura, M. T., Salem, N. Jr., and Moriguchia, T. (2017). Effects of arachidonic acid, eicosapentaenoic acid and docosahexaenoic acid on brain development using artificial rearing of delta-6-desaturase knockout mice. Prost. Leuk. Essen. Fat. Acids 127, 32-39. doi: $10.1016 /$ j.plefa.2017.10.001
Hayashi, A. A., Medeiros, S. R., Carvalho, M. H., and Lanna, D. P. D. (2007). Conjugated linoleic acid (CLA) effects on pups growth, Milk composition and lipogenic and lipogenic enzymes in lactating rats. J. Dairy Res. 74, 160-166. doi: $10.1017 /$ S002202990600224X

Hayat, L., Al-Sughayer, M., and Afzal, M. A. (1999). Comparative study of fatty acids in human breast breast milk substitutes in kuwait. Nutr Res. 19, 827-841. doi: 10.1016/S0271-5317(99)00044-5

Herrera, E. (2002). Implications of dietary fatty acids during pregnancy on placental fetal and postnatal development-a review. Placenta 23, 9-19. doi: 10.1053/plac.2002.0771

Hsieh, T., and Brenna, T. (2009). Dietary docosahexaenoic acid but no arachidonic acid influences central nervous system fatty acid status in baboon neonates. Prost. Leuk. Essen. Fat. Acids 8, 105-110. doi: 10.1016/j.plefa.2009.05.012

Hunt, W. T., Kamboj, A., Anderson, H. D., and Anderson, C. M. (2010). Protection of cortical neurons from excitotoxicity by conjugated linoleic acid. J. Neurochem. 115, 123-130. doi: 10.1111/j.1471-4159.2010.06908

Jelińska, M., Białek, A., Mojska, H., Gielecińsk, I., and Tokarz, A. (2014). Effect of conjugated linoleic acid mixture supplemented daily after carcinogen application on linoleic and arachidonic acidmetabolites in rat serum and induced tumours. Biochim. Biophys. Acta 1842, 2230-2236. doi: 10.1016/j. bbadis.2014.08.013

Leussis, M. P., and Bolivar, V. J. (2006). Habituation in rodents: a review of behavior, neurobiology, and genetics. Neurosci. Biobehav. Res. 30, 1045-1064. doi: 10.1016/j.neubiorev.2006.03.006

Morgane, P. J., Miller, M., Kempler, T., Bronzino, J., Tonkiss, J., Diaz-Cintra, S. et al. (1993). Prenatal malnutrition and development of the brain. Neurosci. Behav. Rev. 17, 91-128. doi: 10.1016/S0149-7634(05)80234-9

Mucci, D. B., Fernandes, F. S., Souza, A. S., Sardinha, F. L. C., Soares-Mota, M., and Carmo, M. G. T. (2015). Flaxseed mitigates brain mass loss, improving motor hyperactivity and spatial memory, in a rodent model of neonatal hypoxicischemic encephalopathy. Prost. Leuk. Essen. Fat. Acids 97, 13-19. doi: 10.1016/ j.plefa.2015.03.001

Muller, C. P., Reichel, M., Mühle, C., Rhein, C., Gulbins, E., and Kornhuber, J. (2015). Brain membrane lipids in major depression and anxiety disorders. Biochim. Biophys. Acta 1851, 1052-1065. doi: 10.1016/j.bbalip.2014 12.014

Pariza, M. W., Park, Y., and Cook, M. E. (2001). The biologically active isomers of conjugated linoleic acid. Prog. Lipid Res. 40, 283-298. doi: 10.1016/S01637827(01)00008-X

Park, Y., Albright, K. J., Storkson, J. M., Liu, W., and Pariza, M. W. (2010). Effects of dietary conjugated linoleic acid (CLA) on spontaneously hypertensive rats. J. Funct. Foods 2, 54-59. doi: 10.1016/j.jff.2010.01.001

Rachetti, A. L. F., Arida, R. M., Patti, C. L., Zanin, K. A., Fernades-Santos, L., Frussa-Filho, R., et al. (2013). Fish oil supplementation and physical exercise program: distinct effects on different memory tasks. Behav. Brain Res. 237 283-289. doi: 10.1016/j.bbr.2012.09.048

Rathod, R., Khaire, A., Kemse, N., Kale, A., and Joshi, S. (2014). Maternal omega-3 fatty acid supplementation on vitamin B12 rich diet improves brain omega-3 fatty acids, neurotrophins and cognition in the Wistar rat offspring. Brain Dev. 36, 853-863. doi: 10.1016/j.braindev.2013.12007

Reeves, P. G., Nielsen, F. H. C., and Fahey, G. C. (1993). AIN-93 purified diets for laboratory rodents: final report of the American Institute of Nutrition. J. Nutr. 123, 939-951. doi: 10.1093/jn/123.11.1939

Rego, O. A., Alves, S. P., Antunes, L. M., Rosa, H. J., Alfaia, C. F., Prates, J. A., et al. (2009). Rumen biohydrogenation-derived fatty acids in milk fat from grazing dairy cows supplemented with rapeseed, sunflower, or linseed oils. J. Dairy Sci. 92, 4530-4540. doi: 10.3168/jds.2009-2060

Ringseis, R., Saal, D., Müller, A., Steinhart, H., and Eder, K. (2004). Dietary conjugated linoleic acids lower the triacylglycerol concentration in the milk of lactating rats and impair the growth and increase the mortality of their suckling pups. J. Nutr. 134, 3327-3334. doi: 10.1093/jn/134.12.3327

Salvati, S., Attorri, L., Avellino, C., Di-Biase, A., and Sanchez, M. (2000). Diet, lipids and brain development. Dev. Neurosci. 20, 481-487. doi: 10.1159/000017479

Salvati, S., Attorri, L., Di Benedetto, R., Di Biase, A., and Leonardi, F. (2006). Polyunsaturated fatty acids and neurological diseases. Mini Rev. Med. Chem. 6, 1201-1211. doi: 10.2174/138955706778742740

Santillan, M. E., Vincenti, L. M., Martini, A. C., Cuneo, M. F., Ruiz, R. D., Mangeaud, A., et al. (2010). Developmental and neurobehavioral effects of 
perinatal expo- sure to diets with different n-6: n-3 ratios in mice. Nutrition 26, 423-431. doi: 10.1016/j.nut.2009.06.005

Shen, P., Kershaw, J. C., Yue, Y., Wang, O., Kim, K. H., McClementsa, D. J., et al. (2018). Effects of conjugated linoleic acid (CLA) on fat accumulation, activity, and proteomics analysis in Caenorhabditis elegans. Food Chem. 249, 193-201. doi: 10.1016/j.foodchem.2018.01.017

Sikorski, A. M., Hebert, N., and Swain, R. A. (2008). Conjugated linoleic acid (CLA) inhibits new vessel growth in the mammalian brain. Brain Res. 40, 35-40. doi: 10.1016/j.brainres.2008.01.096

Smart, J. L., and Dobbing, J. (1971). Vulnerability of developing brain. II. Effects of early nutritional deprivation on reflex ontogeny and development of behavior in the rat. Brain Res. 28, 85-95. doi: 10.1016/0006-8993(71) 90526-9

Soares, J. K. B., Melo, A. P. R., Medeiros, M. C., Queiroga, R. C. R. E., Bomfim, M. A. D., Santiago, E. C. A., et al. (2013). Anxiety behavior is reduced, and physical growth is improved in the progeny of rat dams that consumed lipids from goat milk: Na elevated plus maze analysis. Neurosci. Lett. 552, 25-29. doi: 10.1016/j.neulet.2013.07.028

Soares, J. K. B., Rocha-de-Melo, A. P., Medeiros, M. C., Queiroga, R. C. R. E., Bomfim, M. A. D., Souza, A. F. O., et al. (2012). Conjugated linoleic acid in the maternal diet differentially enhances growth and cortical spreading depression in the rat progeny. Biochim Biophys Acta 1820, 1490-1495. doi: 10.1016/j. bbagen.2012.05.010

Souza, A. S., Rocha, M. S., and Carmo, M. G. T. (2012). Effects of a normolipidic diet containing trans fatty acids during perinatal period on the growth, hippocampus fatty acid profile, and memory of young rats according to sex. Nutrition 28, 458-464. doi: 10.1016/j.nut.2011.08.007
Speight, A., Davey, W. G., McKenna, E., and Voigt, J. P. W. (2017). Exposure to a maternal cafeteria diet changes open-field behaviour inthe developing offspring. Int. J. Dev. Neurosci. 57, 34-40. doi: 10.1016/j.ijdevneu.2016.12.005

Valenzuela, A. B., and Nieto, S. K. (2003). Ácidos grasos omega-6 y omega3 en la nutrición perinatal: su importância em el desarrolo del sistema nervioso y visual. Rev. Child. Pediatr. 74, 149-157. doi: 10.4067/S037041062003000200002

Yu, H., Bi, Y., Ma, W., He, L., Yuan, L., Feng, J., et al. (2010). Long-term effects of high lipid and high energy diet on serum lipid, brain fatty acid composition, and memory and learning ability in mice. Int. J. Dev. Neurosci. 28, 271-276. doi: 10.1016/j.ijdevneu.2009.12.001

Zanarini, M. C., and Frankenburg, F. R. (2003). Omega-3 Fatty acid treatment of women with borderline personality disorder: a double-blind, placebocontrolled pilot study. Am. J. Psychiatry 160, 167-169. doi: 10.1176/appi.ajp. 160.1.167

Conflict of Interest Statement: The authors declare that the research was conducted in the absence of any commercial or financial relationships that could be construed as a potential conflict of interest.

Copyright (c) 2019 Queiroz, Lima, Barbosa, Melo, Bertozzo, Oliveira, Bessa, Alves, Souza, Queiroga and Soares. This is an open-access article distributed under the terms of the Creative Commons Attribution License (CC BY). The use, distribution or reproduction in other forums is permitted, provided the original author(s) and the copyright owner(s) are credited and that the original publication in this journal is cited, in accordance with accepted academic practice. No use, distribution or reproduction is permitted which does not comply with these terms. 